\title{
Acyclovir Induced Acute Kidney Injury In Acute Meningitis Patient: A Case Report Highlights the Concurrence Of AKI Risk Factors And The Neutropenic Effect Of Ticlopidine
}

\author{
Arwa M. Amin ${ }^{1}$, Baharudin Ibrahim ${ }^{2 *}$, Azmi Sarriff ${ }^{3}$ \\ ${ }^{1}$ MPharm Clinical Pharmacy \\ ${ }^{2}$ PhD, MPharm, Senior Lecturer, Discipline of Clinical Pharmacy, email: baharudin.ibrahim@usm.my \\ ${ }^{3}$ PharmD, Associate Professor \& Chairman, Discipline of Clinical Pharmacy \\ ${ }^{1,2,3}$ School of Pharmaceutical Sciences, Universiti Sains Malaysia (USM), 11800 Minden, Penang, Malaysia
}

\begin{abstract}
Nephrotoxicity is one of the challenging side effects of acyclovir use in clinical practice. The Concomitant use of other nephrotoxic antibiotics, in addition to patient's risk factors, can trigger acyclovir induced acute kidney injury (AKI). We described a case of acute meningitis in 68 year old female patient with underlying history of ischemic heart disease managed by ticlopidine. The gram stain, culture and PCR of the CSF did not show any positive growth of bacterial infection or HSV. However, the negative CSF gram stain and culture caused a confusion of the diagnosis of bacterial meningitis with viral meningitis. The patient had AKI induced by the treatment with acyclovir for 16 consecutive days and possibly triggered by other AKI risk factors. Hemodialysis and hydration did not reverse the kidney function to normal. However, it was reversed to its normal status only after the discontinuation of acyclovir. The neutropenic effect of ticlopidine can be a risk to the patient that could lead to fatal infection. In this case report we reviewed the literature on the nephrotoxicity of acyclovir, the neutropenia of ticlopidine and a proposed role of granulocyte colony stimulating factor $(G-$ CSF).
\end{abstract}

KEYWORDS - Acute Kidney Injury, Acyclovir, Meningitis, Neutropenia, nephrotoxicity, granulocyte colony stimulating factor

\section{INTRODUCTION}

The nephrotoxicity of some drugs remains a challenge to the use of these drugs in clinical practices, particularly for critically ill patients or for those who are vulnerable to kidney injury. Acyclovir is an anti-viral drug which is used in the management of some of the central nervous system (CNS) viral infections [1]. Acyclovir nephrotoxicity has been documented [2-4]. Acute kidney injury (AKI) induced by acyclovir could be due to intratubular crystal precipitation or direct insult to renal tubular cells $[4,5]$. Yet, the co-administration of acyclovir with other nephrotoxic drugs, such as aminoglycosides and vancomycin, can accelerate the occurrence of AKI [6-9]. This requires careful monitoring while prescribing nephrotoxic anti-microbial therapy. Infectious meningitis can be due to bacterial, viral, fungal, mycobacterium and parasites [10]. Except for herpes simplex virus infections and meningoencephalitis, viral meningitis can be managed by supportive treatment without major consequences [11]. The analysis of cerebrospinal fluid (CSF) remains the best method to diagnose meningitis accurately where the imperative sign of meningitis in CSF analysis is pleocytosis [12]. However, sometimes the CSF analysis does not provide definite indication of the microbial infection causing meningitis. It is crucial to start the empirical treatment of bacterial meningitis based on patients' age and risk factors before obtaining the confirmatory CSF lab results to avoid any squalled due to the delay of treatment [12]. In this case report, we will describe a case of acyclovir induced AKI which was triggered by the concomitant use of amikacin and vancomycin in an acute infectious meningitis patient. The patient was treated with acyclovir for 16 consecutive days and did not recover from AKI until acyclovir was discontinued.

\section{CASE REPORT}

A 68 year old Malaysian Chinese woman was referred to the hospital after an initial diagnosis of acute bacterial meningitis. This diagnosis was made by another hospital near to her house. The patient had a medical history of hypertension, dyslipidemia and hypothyroidism. She was allergic to penicillin. A year ago, she was diagnosed with ischemic heart disease (IHD) and had coronary magnetic angiogram and angioplasty. Initially she was on daily antiplatelet therapy of $75 \mathrm{mg}$ clopidogrel tablets. However, upon her request for cheaper antiplatelet, it was changed to $250 \mathrm{mg}$ ticlopidine tablets twice daily since two months ago. 
Her other medications included $2 \mathrm{mg}$ perindopril tablets to be taken once daily, $10 \mathrm{mg}$ amlodipine tablets $10 \mathrm{mg}$ once daily, $40 \mathrm{mg}$ simvastatine tablets once every night, $25 \mathrm{mg}$ metoprolol tablets twice daily and $100 \mu \mathrm{g}$ levothyroxine tablets once daily. She lives with her husband and claimed to be free of smoking and alcohol drinking. Three days after returning from a journey to Thailand, she had fever and an episode of seizures. She went to a nearby hospital where they suspected that she suffered from meningitis, and had the first lumbar puncture (LP) for CSF analysis. The results showed elevated protein and slight pleocytosis; the red blood cells (RBCs) were 15 cells $/ \mu \mathrm{L}$, the white blood cells (WBCs) were 5 cells $/ \mu \mathrm{L}$, the protein level was 1.17 $\mathrm{g} / \mathrm{L}$ and the glucose was $3.1 \mathrm{mmol} / \mathrm{L}$ (reference range $(2.2-4.2 \mathrm{mmol} / \mathrm{L}$ ) and blood glucose was $4.2 \mathrm{mmol} / \mathrm{L}$ ). The CSF gram stain, culture, acid fast bacilli and Indian ink wet preparation for cryptococcus was negative, and there was no growth. The patient was empirically treated with intravenous (I.V.) ceftriaxone $2 \mathrm{~g}$ twice daily in addition to I.V. phenytoin $100 \mathrm{mg}$ every eight hours and intravenous continuous infusion of midazolam.

Upon her current admission, the patient was in acute confusion state. Her body temperature was $37.8^{\circ} \mathrm{C}$, the blood pressure was $153 / 75 \mathrm{mmHg}$ and the pulse rate was $90 /$ minutes. The blood profile showed WBCs $\left(10.0 \times 10^{3} / \mu \mathrm{L}\right)$, the relative neutrophils count was slightly low $(49.6 \%)$, RBCs $\left(4.5 \times 10^{6} / \mu \mathrm{L}\right)$, hemoglobin of $13.3 \mathrm{~g} / \mathrm{dL}$ and platelet count of $169 \times 10^{3} / \mu \mathrm{L}$. The patient renal function on admission was normal with creatinine level of $49 \mu \mathrm{mol} / \mathrm{L}$ and calculated creatinine clearance of $99.73 \mathrm{ml} / \mathrm{min}$. The alanine aminotransferase (ALT) and alkaline phsosphatase (ALP) were normal but she had a slightly low total amount of protein $(63 \mathrm{~g} / \mathrm{L})$ and albumin $(30 \mathrm{~g} / \mathrm{L})$. Her thyroid stimulating hormone level was $0.18 \mathrm{mlU} / \mathrm{L}$ and thyroxine was $(22.2 \mathrm{pmol} / \mathrm{L})$. The blood viruses tests showed negative HIV1, non-reactive for both hepatitis B surface antigen (HBsAg) and hepatitis C antibody (HCV-3). A computed tomography (CT) scan of the brain indicated cerebral atrophy and the absence of intercerebral hemorrahge. The electro encephalogram (EEG) suggested that there was severe diffuse encephalopathy.

The patient's initial empiric treatment with ceftriaxone was continued for two more days to complete four days of treatment which was started by the previous hospital. Then it was changed to I.V. $2 \mathrm{~g}$ meropenem and 500mg acyclovir, both every 8 hours. This therapy continued for 16 days. Although being on antimicrobial therapy, the patient's temperature continued to rise up to $39.3^{\circ} \mathrm{C}$, so I.V. $750 \mathrm{mg}$ amikacin once daily was added on day three (D3). Due to the continuous increase of her temperature and as methicillin resistant staphylococcus aureus (MRSA) had been reported before in the same ward, she was suspected of MRSA and she was given I.V. vancomycin $750 \mathrm{mg}$ once daily on day four (D4). After three days of acyclovir therapy and one day after adding amikacin then vancomycin, it was discovered that the serum creatinine level had increased to $255 \mu \mathrm{mol} / \mathrm{L}$ and continued to increase to $364 \mu \mathrm{mol} / \mathrm{L}$. In addition, her calculated creatinine clearance had reduced to $13.4 \mathrm{ml} / \mathrm{min}$. Then, both amikacin and vancomycin were stopped immediately, except for acyclovir. In spite of the discontinuation of the two drugs, creatinine level still continue to increase reaching $450 \mu \mathrm{mol} / \mathrm{L}$ and the calculated creatinine clearance was $10.7 \mathrm{ml} / \mathrm{min}$. Subsequently, she developed metabolic acidosis and septic shock. A diagnosis of sepsis due to drug induced acute kidney injury (AKI) was established and the cause was referred to be due to both amikacin and vancomycin. The patient was transferred to the intensive care unit (ICU). She was given adequate hydration and had hemodialysis for two times. In spite of the indicated AKI, acyclovir treatment was still continued for 16 days. It was discovered that the creatinine level continued to be persistently elevated $(330-225 \mu \mathrm{mol} / \mathrm{L})$. When acyclovir was stopped, after two days, the creatinine level had reduced to $93 \mu \mathrm{mol} / \mathrm{L}$. It continued to be reduced further to $49 \mu \mathrm{mol} / \mathrm{L}$ after a week of the discontinuation of acyclovir. The patient's body temperature, renal function and physical status were found to be improved.

A second LP and CSF analysis was made after ten days of her admission which showed the reduction of both protein $(0.8 \mathrm{~g} / \mathrm{L})$ and WBCs $(3$ cells $/ \mu \mathrm{L})$. However, the glucose had increased to $5.3 \mathrm{mmol} / \mathrm{L}$. Again, the CSF gram stain, culture, acid fast bacilli and Indian ink wet preparation for cryptococcus showed to be negative and no growth. A CSF sample was sent for polymerase chain reaction assay (PCR) of herpes simplex virus (HSV1) and the result was found to be negative. In addition her antimicrobial therapy, the patient was given I.V. $250 \mathrm{mg}$ leviteracitam twice daily for two weeks and I.V. 100mg phenytoin every eight hours for the seizure. The patient's seizures were well controlled and she did not experience any episode of it. The patient continued to take her IHD, hypertension, dyslipidemia and hypothyroidism medications until she developed sepsis and they were discontinued. Except for ticlopidine which was replaced by clopidogrel, the rest of her medications were restarted after her recovery from sepsis. The patient continued to improve after the completion of 16 days of treatment with meropenem and the discontinuation of acyclovir and then ticlopidine. Her liver profile, kidney function and blood cell count all become normal on day 26 of her admission. Her full recovery was after thirty days of admission and she was discharged. 


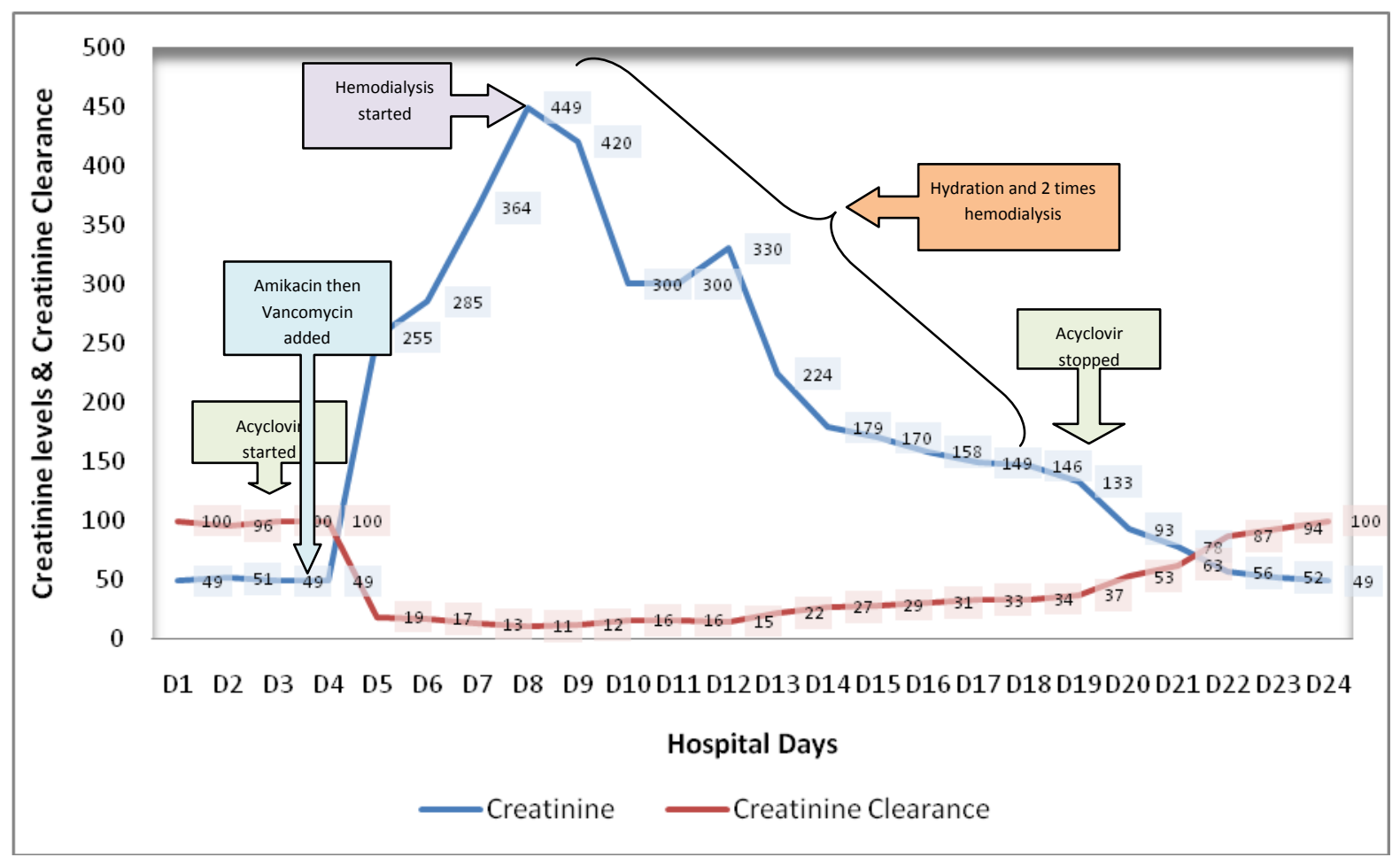

Figure 1: Creatinine levels $(\mathrm{Cr})$ and creatinine clearance $(\mathrm{Cr} \mathrm{Cl})$ of the patient during 24 days of her hospital stay

\section{DISCUSSION}

Although acyclovir is a crucial treatment of herpes simplex and varicella zoster viruses [1], its induced nephrotoxicity requires judicious decision before using it. Acyclovir induced nephrotoxicity is believed to be due to intra-tubular precipitation of crystals [4]. However, recent reports showed that it could be due to the direct insult to tubular cells [5]. This requires critical prescribing, effective hydration and extensive monitoring of renal function. There are several risk factors which can aggravate the renal insult of nephrotoxic drugs, such as patient's age (> 65 years), female sex, impaired immunity, the duration of using the nephrotoxic drug and concurrent use of other nephrotoxic drugs $[6,7,9,13]$. Acyclovir induced nephrotoxicity can occur within 1272 hours after starting the therapy $[4,14]$. This nephrotoxicity can lead to AKI if it was not early identified and the patient may consequently require hemodialysis $[4,14]$. The fast increase in serum creatinine is an important indicator of acyclovir nephrotoxicity [15]. To lower the risk of acyclovir nephrotoxicity, hydration with isotonic fluids during the administration of the drug is imperative [14]. The cause of AKI in this patient was referred to be due to amikacin and vancomycin as the deterioration of renal function occurred after their addition to the therapy.

Vancomycin induced nephrotoxicity usually arise after the prolonged use of it for more than 7 days [7, 8]. However, in this patient, she was given vancomycin for one dose only and then it was discontinued. The incidence of amikacin induced nephrotoxicity usually occur within 4 to 5 days after starting the therapy [8], which also was not the case of this patient. However, the co-administration of vancomycin and amikacin can accelerate the occurrence of AKI [8,9]. Despite the discontinuation of both amikacin and vancomycin, adequate hydration and the hemodialysis, the renal function of the patient continued to decline with the continuation of acyclovir. This could be due to the development of AKI and the patient might had direct tubular insult which requires a prompt discontinuation of the drug. It is also worthy to note, that this patient was given ceftriaxone for 4 days before replacing it with meropenem. Ceftriaxone can also cause nephrotoxicity [8]. Therefore, due to the continuous deterioration of the renal function though the discontinuation of amikacin and vancomycin in this patient, we can infer that acyclovir could be the initial cause of nephrotoxicity and the presence of several risk factors could had led to the development of AKI. The concurrent use of other nephrotoxic drugs before and after starting acyclovir, the patient's age (68 years) and sex (female), all together increased the renal vulnerability to develop AKI. As the first CSF analysis PCR assay of HSV1 was negative and the second analysis after one week of acyclovir treatment was negative as well, it was optimum to promptly discontinue acyclovir and to continue the hydration treatment. 
Factors such as age, immunity status of the patient, the recent history of neurosurgery and the season of the infection can help to determine the type of pathogens that cause meningtis. In adults of more than 50 year old, Streptococcus pneumoniae, Neisseria meningitidis, Listeria monocytogenes and aerobic gram negative bacilli are the common pathogens [12]. Viral meningitis is usually caused by enterovirus, herpes simplex virus (HSV), and varicella-zoster virus [10]. Cryptococcal meningitis and Mycobacterium tuberculosis are common in immunodeficient patients such as HIV patients [16, 17]. In bacterial meningitis, CSF analysis usually shows white blood cells $(\mathrm{WBCs}) \geq 100 / \mu \mathrm{l}$ with predominance of neutrophils, normal glucose and protein $\geq 0.5 \mathrm{~g} / \mathrm{L}[10$, 12]. The positive results of bacterial growth culture and gram stain asserts the pathogenic cause of meningitis. However, in many cases the cut off point of diagnosis is not obvious since there may be many factors that may interfere. In this report, the patient's CSF analysis did not show positive bacterial gram stain and culture. In a study conducted on 297 adults with suspected meningitis, only $1 \%$ had been confirmed to have bacterial meningitis based on their CSF lab results [18]. The magnetic resonance imaging (MRI) is the preferred imaging tool to aid CSF-PCR assay in indicating HSV encephalitis [19]. Some reported cases revealed that MRI is significant in the negative results of CSF-PCR assay [20]. Although the CT and EEG showed some abnormalities in this case, the MRI was not done for the patient on her initial presentation. This hindered the definite diagnosis, thereby leading to the suspicion of herpes simplex encephalitis (HSE). Due to the unclear diagnosis of meningitis aetiology in this patient, acyclovir in addition to meropenem continued to be administered for 16 consecutive days.

The use of ticlopidine antiplatelet was reduced in the last decade due to its side effects such as neutropenia, thrombotic thrombocytopenic purpurea and liver toxicity [21-25]. In some cases, the occurrence of neutropenia can appear after eight weeks from initiation $[25,26]$. It was reported that the neutropenic effect of ticlopidine had led to fatal outcome in patients with infectious diseases [27, 28]. This patient was having mild systemic neutropenia which might be due to the prior use of ticlopidine. The confirmation of her neutropenic extent was difficult because there was no record of her neutrophils pre ticlopidine use. Clopidogrel, which is the second generation thienopyridine antiplatelet, is safer, less neutropenic and less hepatotoxic [21]. It was imperative to replace ticlopidine with clopidogrel. The patient should be warned against the consequences of any future replacement of clopidogrel with ticlopidine.

The Neupogen ${ }^{\circledR}$ (Filgrastim), which is synthesized recombinant protein of the endogenous granulocyte stimulating factor (G-CSF), could have role in the management of bacterial meningitis. It is being used to overcome neutropenia induced by chemotherapy [29] and other drugs such as ticlopidine [23, 30]. By increasing the proliferation of leukocytes, G-CSF will lead to better management of systemic infections and hence will help patients to recover from CNS infections [31]. This adjunctive role of G-CSF in treating infections and sepsis was investigated in several studies [32-36]. A study in pneumococcal meningitis revealed encouraging results [36]. A daily dose of subcutaneous (S.C.) $300 \mu \mathrm{g}$ G-CSF for 6 days, cefotaxime 9-12 g/day for 10 days and dexamethasone $16 \mathrm{mg}$ /day for 3 days in 22 non-neutropenic patients proved to be effective and safe [36]. The results showed rapid recovery of indices of CSF inflammation, neurological status and an improvement in CSF glucose level. Further large scale studies on G-CSF role in infectious meningitis are required to assert its adjunctive role.

\section{CONCLUSION}

In conclusion, the use of acyclovir, particularly for prolonged therapy, should be considered with care of the risk of nephrotoxicity so as to avoid AKI. Concomitance use of nephrotoxic drugs should be avoided especially in elderly patients and those with renal impairment. Unless it is being diagnosed of HSE meningitis, viral meningitis in adults is self-treated and the prolonged administration of acyclovir is not required. The blood count of patients on thienopyridine antiplatelet, particularly ticlopidine, has to be checked periodically to early detect any blood discrepancies. The use of adjunctive G-CSF therapy to treat infectious diseases, including meningitis, might be substantial but needs further investigations.

\section{REFERENCES}

[1] Gunness, P., et al., Acyclovir-induced nephrotoxicity: the role of the acyclovir aldehyde metabolite. Translational research : the journal of laboratory and clinical medicine, 2011. 158(5): p. 290-301.

[2] Fleischer, R. and M. Johnson, Acyclovir nephrotoxicity: a case report highlighting the importance of prevention, detection, and treatment of acyclovir-induced nephropathy. Case Rep Med, 2010. 2010.

[3] Obada, E.N., et al., [Acute renal failure following a treatment with acyclovir]. Nephrol Ther, 2010. 6(2): p. 125-7.

[4] Seedat, A. and G. Winnett, Acyclovir-induced acute renal failure and the importance of an expanding waist line. BMJ Case Rep, 2012. 2012.

[5] Gunness, P., et al., Acyclovir-induced nephrotoxicity: the role of the acyclovir aldehyde metabolite. Transl Res, 2011. 158(5): p. 290301.

[6] Oliveira, J.F.P., et al., Prevalence and Risk Factors for Aminoglycoside Nephrotoxicity in Intensive Care Units. Antimicrobial Agents and Chemotherapy, 2009. 53(7): p. 2887-2891. 
[7] Elyasi, S., et al., Vancomycin-induced nephrotoxicity: mechanism, incidence, risk factors and special populations. A literature review. European Journal of Clinical Pharmacology, 2012. 68(9): p. 1243-1255.

[8] Khalili, H., S. Bairami, and M. Kargar, Antibiotics induced acute kidney injury: incidence, risk factors, onset time and outcome. Acta Med Iran, 2013. 51(12): p. 871-8.

[9] Gupta, A., M. Biyani, and A. Khaira, Vancomycin nephrotoxicity: myths and facts. Neth J Med, 2011. 69(9): p. 379-83.

[10] Bamberger, D.M., Diagnosis, initial management, and prevention of meningitis. Am Fam Physician, 2010. 82(12): p. 1491-8.

[11] Kupila, L., et al., Etiology of aseptic meningitis and encephalitis in an adult population. Neurology, 2006. 66(1): p. 75-80.

[12] Tunkel, A.R., et al., Practice guidelines for the management of bacterial meningitis. Clin Infect Dis, 2004. 39(9): p. 1267-84.

[13] Perazella, M.A., Renal vulnerability to drug toxicity. Clin J Am Soc Nephrol, 2009. 4(7): p. 1275-83.

[14] Kim, S. and Y. Byun, Comparison of Renal Function Indicators According to Hydration Volume in Patients Receiving Intravenous Acyclovir With CNS Infection. Biological Research For Nursing, 2014.

[15] Yildiz, C., et al., Acute kidney injury due to acyclovir. CEN Case Reports, 2013. 2(1): p. 38-40.

[16] Park, B.J., et al., Estimation of the current global burden of cryptococcal meningitis among persons living with HIV/AIDS. AIDS, 2009. 23(4): p. 525-530 10.1097/QAD.0b013e328322ffac.

[17] Török, M.E., et al., Timing of Initiation of Antiretroviral Therapy in Human Immunodeficiency Virus (HIV)-Associated Tuberculous Meningitis. Clinical Infectious Diseases, 2011. 52(11): p. 1374-1383.

[18] Thomas, K.E., et al., The diagnostic accuracy of Kernig's sign, Brudzinski's sign, and nuchal rigidity in adults with suspected meningitis. Clin Infect Dis, 2002. 35(1): p. 46-52.

[19] Tyler, K.L., Update on herpes simplex encephalitis. Rev Neurol Dis, 2004. 1(4): p. 169-78.

[20] Adler, A.C., et al., Herpes simplex encephalitis with two false-negative cerebrospinal fluid PCR tests and review of negative PCR results in the clinical setting. Case Rep Neurol, 2011. 3(2): p. 172-8.

[21] Maseneni, S., et al., Toxicity of clopidogrel and ticlopidine on human myeloid progenitor cells: Importance of metabolites. Toxicology, 2012. 299(2-3): p. 139-145.

[22] Paradiso-Hardy, F.L., et al., Hematologic dyscrasia associated with ticlopidine therapy: evidence for causality. Cmaj, 2000. 163(11): p. 1441-8.

[23] Previtera, A.M. and R. Pagani, Agranulocytosis and hepatic toxicity with ticlopidine therapy: a case report. 2010.

[24] Ceylan, C., et al., Early ticlopidine-induced hepatic dysfunction, dermatitis and irreversible aplastic anemia after coronary artery stenting. Am J Hematol, 1998. 59(3): p. 260.

[25] Fukushima, K., et al., Incidence of side-effects of ticlopidine after sirolimus-eluting stent implantation. Circ J, 2007. 71(4): p. 617-9.

[26] Farver, D.K. and L.A. Hansen, Delayed neutropenia with ticlopidine. Ann Pharmacother, 1994. 28(12): p. 1344-6.

[27] Szto, G.Y., T.J. Linnemeier, and M.W. Ball, Fatal neutropenia and thrombocytopenia associated with ticlopidine after stenting. Am J Cardiol, 1999. 83(1): p. 138-9.

[28] Geletko, S.M., K.M. Melbourne, and D.J. Mikolich, Pseudomonas bacteremia precipitated by ticlopidine-induced neutropenia. Ann Pharmacother, 1996. 30(3): p. 246-8.

[29] Welte, K., et al., Filgrastim ( $r$-metHuG-CSF): the first 10 years. Blood, 1996. 88(6): p. 1907-29.

[30] Andres, E. and F. Maloisel, Idiosyncratic drug-induced agranulocytosis or acute neutropenia. Curr Opin Hematol, 2008. 15(1): p. 1521.

[31] Mook-Kanamori, B.B., et al., Pathogenesis and pathophysiology of pneumococcal meningitis. Clin Microbiol Rev, 2011. 24(3): p. $557-91$.

[32] Sundar, K.M. and M. Sires, Sepsis induced immunosuppression: Implications for secondary infections and complications. Indian J Crit Care Med, 2013. 17(3): p. 162-169.

[33] Carr, R., N. Modi, and C. Dore, G-CSF and GM-CSF for treating or preventing neonatal infections. Cochrane Database Syst Rev, 2003(3): p. Cd003066.

[34] Cheng, A.C., D.P. Stephens, and B.J. Currie, Granulocyte colony stimulating factor (G-CSF) as an adjunct to antibiotics in the treatment of pneumonia in adults. Cochrane Database Syst Rev, 2003(4): p. Cd004400.

[35] Cruciani, M., et al., Granulocyte-colony stimulating factors as adjunctive therapy for diabetic foot infections. Cochrane Database Syst Rev, 2013. 8: p. Cd006810.

[36] de Lalla, F., R. Nicolin, and L. Lazzarini, Safety and efficacy of recombinant granulocyte colony-stimulating factor as an adjunctive therapy for Streptococcus pneumoniae meningitis in non-neutropenic adult patients: a pilot study. J Antimicrob Chemother, 2000. 46(5): p. 843-6. 